\title{
Comparison of Radio Wave Propagation Models for Mobile Networks
}

Aigerim Bakatkaliyevna Altayeva and Young Im Cho

Department of Computer Engineering, Gachon University, Seongnam, Korea

\begin{abstract}
Heterogeneous cellular networks are gaining momentum in industry and the research community, and are attracting the attention of standard bodies such as 3GPP LTE and IEEE 802.16j, whose objectives are to increase the capacity and coverage of cellular networks. In this article, we provide an overview of expansion strategies, optimal locations of base stations with different characteristics, and radio-planning models.
\end{abstract}

Keywords: 3G, 4G, LTE, MIMO, Base station

\section{Introduction}

The successful operation of modern cellular mobile communication networks depends not only on initial planning, but also on adaptability to changing conditions. In large cities with developed infrastructure, the constantly changing environment requires the cellular operator to quickly react to environmental changes. Permanently increasing the volume of transmitted information requires optimization of honeycomb network configuration, the search for optimal methods for electromagnetic compatibility(EMC), and spatial frequency resource distribution for continuous monitoring of overloads. Highly effective networks operators planning, optimization, and operation of the network using expensive software using models of propagation of electromagnetic waves in various conditions, taking into account terrain based geo-information technology(GIS) and EMC control [1,2]. In the field of mobile communication network planning methods are available as frequency-territorial coverage and service areas forecasts (i.e., of coverage) for base stations (BSs).

Received: Aug. 24, 2015

(c) This is an Open Access article distributed under the terms of the Creative Commons Attribution Non-Commercial License (http://creativecommons.org/licenses/ by-nc/3.0/) which permits unrestricted noncommercial use, distribution, and reproduction in any medium, provided the original work is properly cited.

\section{Scheduling in Base Station Cellular Networks}

The main task when planning mobile networks is to provide an adequate frequency plan and the necessary coverage with high quality and minimal investment in a given region. Despite the existence of well-known algorithms for constructing networks, the planning process and criteria vary depending on the relevant dominant factors and are individually selected for each task.

In general network planning is an iterative process that includes the following steps:

(1) Synthesis of the network structure;

(2) Prediction of the signal field strength within the network;

(3) Analysis of the service area of each cell and the entire network; 
(4) Assessment of the intra electromagnetic compatibility;

(5) Frequency assignment;

(6) Analysis of the functioning of the network, taking into account interference.

The iterative planning process continues until a radio network $S^{\prime \prime}$ satisfying the original requirements (constraints) with quality $\bar{K}\left(S^{\prime \prime}\right)$ is found, which is optimized in the sense of unconditional preference criterion, compared with $\bar{K}\left(S^{\prime}\right)$ :

$$
\bar{K}\left(S^{\prime \prime}\right)<\bar{K}\left(S^{\prime}\right)
$$

The initial set of feasible options for the structure and placement of base stations, the set of performance indicators $E_{n}=$ $\left\{E_{1}, \ldots, E_{k}\right\}$ and their weights $z_{i}(i=\overline{1, k})$ should be determined based on the preferences of the customer.

The sequence of actions when planning a mobile network are as follows:

(1) Preparation of raw data.

(2) Calibration of mathematical propagation models based on the measurements of the field strength in the most characteristic point within the network coverage.

(3) Construction of a first approximation of the radio network.

(4) Locating sites deploying base stations in the countryside and iterative optimization with extensive use of funds software.

The most useful approach to solving this problem is decomposition of the planning process into several stages. When this approach is applied to the problem of frequency territorial planning projects, it provides a network based on customer preferences that can be used as a reference. The main stages of frequency-spatial planning are described below.

The first stage involves preparing digital topographic maps of the area, which contain data on terrain, the development of the territory, and the locations of forests and water bodies.

The second stage consists of obtaining data regarding terrain height, land use, distributions of traffic flows, and other factors affecting signal density

Additionally, the intensity of traffic, forecasts of the number of subscribers, recommended sites for base stations, available bandwidth, compatibility with other systems, and network interfaces should be considered [3].
The third stage begins with the construction of the original radio network $S^{\prime}$. This procedure reduces to determining the location of the BSs, their parameters, and their frequency resource allocation which is based on an assessment of traffic intensity and the nature of the terrain, the selection of the clusters, a preliminary assessment of the BS coverage areas, and the calculation of technical parameters of the BS (including transmitter power, suspension height, and the azimuthal and tilt angles of antennas)

In the fourth step, the binding sites deploying BSs are defined in the plan to build the network areas and perform iterative optimization of geo-information databases using special software.

In this paper, we will consider the following processes: searching sites for base stations, performing a preliminary assessment of the BS coverage areas, and calculating the technical parameters of the BS (transmitter power, suspension height, and azimuthal and tilt angles of antennas).

Note that there is a high mutual influence of these items on each other. Initially, the process starts with finding sites for the BS. The site selection criteria are forecasted intensity workloads and continuous coverage in the planned area, which are determined by the number and type of BS cluster. The result of the process described above is a provisional plan for the deployable communications network.

Studies of load (traffic) have received much attention because design errors can be costly. The plan designed by placing the BSs must identify (and then predict) the pre-service area and create the network frequency plan $[4,5]$.

Under the condition of having limited frequency resources, frequency reuse in the selected frequency range allows for continuous RF coverage over large areas. In the classical theory of cell planning, cells are grouped into clusters. Each cluster uses a fixed set of frequencies that will be repeated after a certain distance. In addition to providing continuous coverage over large areas, frequency reuse in cellular networks increases the capacity of the system.

The possibility of reuse of given frequency determines the high frequency spectrum utilization in cellular systems. Frequencies are allocated within the cluster such that they minimize interference with adjacent channels [6]. These considerations constitute an initial planning phase for the network. Problem areas of unaccounted swampiness and the final network configuration are then determined. These configurations have been in operation since their initial development, i.e., launching the network in commercial operation. 


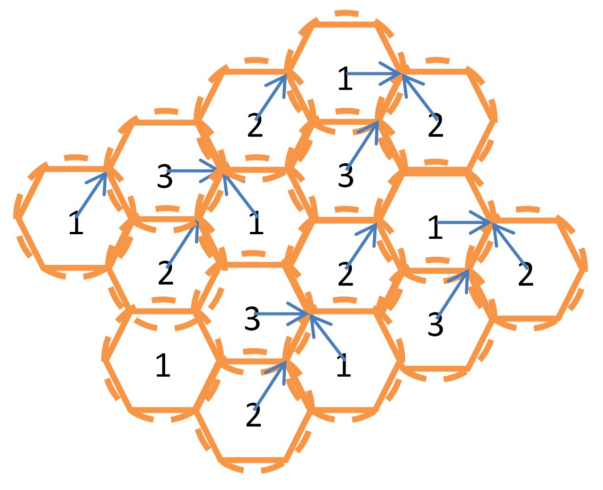

Figure 1. Homogenous base station planning structure.

\section{Cellular Network Planning}

During deployment, there can be problems such as BS location problem, frequency planning, and radio resource management. In this study, the authors consider the BS location problem. In any wireless network planning problem, the radio model is a key component. In theory, radio models can be arbitrarily complex. Working with such models, it is important to find a suitable level of abstraction, i.e., capturing a small number of characteristics may result in a shorter computational time but poor results, and vice versa [6].

Mobile phone systems provide telecommunication services using a set of BSs that can create radio connections with mobile stations (MSs) within their service area. Each service area, called a cell, is a set of points at which the intensity of the signal received from the $\mathrm{BS}$ under consideration is higher than that received from the other BSs. Cells can have different shapes and sizes depending on the BSs location, configuration parameters, and propagation properties [7].

Determining the BS locations is a fundamental planning task. Because of the increasing number of subscribers and increasing user demand, many more and more stations are necessary. The result of the optimization problem is used to determine the BS characteristics [8] and propose optimal geometric structures that minimize both interference and the number of uncovered locations.

When planning a radio network, one implementation uses a homogenous structure of BSs [9-11]. An illustration of these structure is shown in Figure 1. In practice, factors such as the lie of the ground, subscribers' density, automobile noise, the height of the buildings necessitate the use of a heterogeneous structure of BSs.

However, problem can arise when installing BSs with dif-

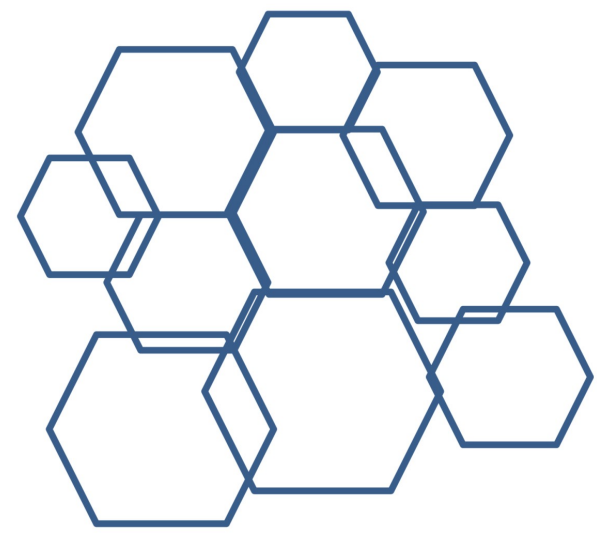

Figure 2. Heterogeneous base station planning structure.

ferent structures. During the planning of heterogeneous BSs, additional interference or uncovered areas can occur, as shown in Figure 2. Our goal is to offer a solution to these problem(i.e., to minimize interference and prevent uncovered areas).

BS locations, another vital issue is frequency assignment. To avoid interference among new mobile technologies, neighboring cells should use different frequencies [12]. In Figure 1 and 2 , the number in the center of the clusters indicates the frequency.

\subsection{Heterogeneous Networks and Their Requirements}

Heterogeneous networks are being used in a variety of areas: cellular telecommunications is a high-profile example, but they are also being used in many other areas, particularly where ad-hoc networks are being deployed. The network must be structured in such a way that the performance, as seen in all areas of the network, meets the requirements. Aspects such as latency, performance and speed must meet the requirements throughout the network[1].

With the challenges facing operators increasing user demanding for improved performance and lower costs, operators will be required to use an ever wider array of technologies to ensure satisfactory operation of their networks in a variety of scenarios.

To provide not only coverage, but the correct form of coverage in terms of small and macro cells, operators deploy a variety of types of base station; they must also implement a heterogeneous format for the backhaul.

With over $60 \%$ of traffic occurring within urban areas it is necessary to deploy a blend of macro cells and metro(i.e., small) cells in the environment. Because city planners want to keep the visual impact to a minimum, towers and masts are much less 
desirable for use in base stations. Unobtrusive small cells will be deployed alongside Wi-Fi to provide the required coverage. All of these systems must operate seamlessly as far as the user is concerned[2].

\subsection{Radio Wave Propagation Models}

Simulation of propagation is based on the prediction average received signal level at a given distance from the emitter; its dispersion values are based on the situation in the radio communication area. The radio coverage area of the transmitter can be determined by calculations. Simulation of the average signal based on the distance between the transmitter and the receiver is called large-scale simulation, because it allows one to determine the signal over long distances (several hundred or thousand feet). On the other hand, the other model is characterized by rapidly changing the values of the received signal level at small displacements (several wavelengths), or for a short time (several seconds); these are called small-scale models.

A model of wave propagation in free space is used to calculate the received signal in the case of the transmitting and receiving antennas located in a radio communication area free of obstacles. This model is used to analyze radio communication via satellite and terrestrial radio links operating at microwave frequencies (3 to $30 \mathrm{GHz}$ ).

When a radio wave propagates in free space, the output power of the receiving antenna, which is expressed as a function of the distance to the transmitting antenna, and the gain of the transmitting and receiving antenna.

When using this equation, it is assumed that the receiving antenna is located at a distance from the transmitter that corresponds to the far field (the Fraunhofer zone).

The space equation is often expressed in relation to a reference point located in the Fraunhofer zone. The reference distance is usually chosen to be $100 \mathrm{~m}$ or $1 \mathrm{~km}$ for communication over open countryside.

One of the most important characteristics of the communication channel is its signal propagation attenuation. The free space attenuation (in $\mathrm{dB}$ ) in the Fraunhofer zone is determined by the expression

A different form of convenient attenuation in free space is in equation (2):

$$
P_{r}(d)=\frac{P_{t} G_{t} G_{r} \lambda^{2}}{(4 \pi)^{2} d^{2} L}
$$

where $P_{t}$ is the transmitted power, $P_{r}(d)$ is the received power, $G_{t}$ is the transmitter antenna gain, $G_{r}$ is the receiver antenna gain, $d$ is the Tx-Rx separation and $L$ is the system loss factor depended upon line attenuation, filter losses and antenna losses and not related to propagation. The gain of the antenna is related to the effective aperture of the antenna which in turn is dependent upon the physical size of the antenna as given the following equation (3).

$$
G=4 \pi A_{e} / \lambda^{2}
$$

The path loss, representing the attenuation suffered by the signal as it travels through the wireless channel is given by the difference of the transmitted and received power in $\mathrm{dB}$ and is expressed as:

$$
P L(d B)=\frac{10 \log P_{t}}{P_{r}}
$$

The fields of an antenna can broadly be classified in two regions, the far field and the near field. It is in the far field that the propagating waves act as plane waves and the power decays inversely with distance. The far field region is also termed as the Fraunhofer region and the Friis equation holds in this region. Hence, the Friis equation is used only beyond the far field distance, $d_{f}$, which is dependent upon the largest dimension of the antenna as the following equation (5).

$$
d_{f}=\frac{2 D^{2}}{\lambda}
$$

Also we can see that the Friis equation is not defined for $d=0$. For this reason, we use a close in distance, $d_{o}$, as a reference point. The power received, $P_{r}(d)$ is then given by:

$$
P_{r}(d)=P_{r}\left(d_{o}\right)\left(d_{o} / d\right) 2
$$

Propagation models that estimate the average signal power for different distances between transmitter and receiver, from several hundred to several thousand meters, are called largescale propagation models. Large-scale models are very simple and do not take into account very small changes, such as attenuation due to multipath propagation. These models are useful for predicting the coverage of radio systems[1,2].

\subsubsection{The Okumura model}

The Okumura model is based on a graphical representation of experimental data obtained for signal level measurement in Tokyo. This experimental approach is a widely used method for the calculation of radio information in a city. The Okumura model is used for the parameter values in the range indicated in 
Table 1. Parameter values for the Okumura model

\begin{tabular}{|c|c|c|}
\hline \multirow[t]{2}{*}{ Parameters } & \multicolumn{2}{|c|}{$\begin{array}{l}\text { Areas of application the } \\
\text { Okumura model }\end{array}$} \\
\hline & Base & Extended \\
\hline $\begin{array}{l}\text { Operating frequency } \\
(\mathrm{MHz})\end{array}$ & $\begin{array}{l}\text { from } 150 \text { to } \\
2000\end{array}$ & to 3000 \\
\hline Length highway $(\mathrm{km})$ & from 1 to 20 & to 100 \\
\hline BS antenna height $(\mathrm{m})$ & $\begin{array}{l}\text { from } 30 \text { to } \\
400\end{array}$ & $\begin{array}{l}\text { from } 1 \sim 5 \text { to } \\
1000\end{array}$ \\
\hline $\begin{array}{l}\text { Antenna height speakers } \\
\text { (m) }\end{array}$ & from 1 to 10 & from 1 to 10 \\
\hline
\end{tabular}

BS, base station.

Table 1 and Figure 3.

The Okumura model based on the results of experimental studies (and compared with the two-beam model) can more accurately predict the average value of the attenuation of radio signals at a relatively long distance between the transmitting and receiving antennas (over $1 \mathrm{~km}$ ) in the presence of obstacles.

According to the model, the average attenuation in $\mathrm{dB}$ is defined. Formula for Okumura model is expressed below:

$L_{m}(d B)=L_{F}(d)+A_{m u}(f, d)-G\left(h_{r}\right)-G\left(h_{t}\right)-G_{A R E A}$

Where;

$L_{m}=$ (i.e., median) of path loss

$L_{F}(d)=$ free space propagation path loss.

$A_{m u}(f, d)=$ median attenuation relative to free space

$G\left(h_{b}\right)=$ base station antenna height gain factor

$G\left(h_{m}\right)=$ mobile antenna height gain factor

$G\left(h_{b}\right)=20 \log \left(\mathrm{h}_{b} / 200\right) 1000 \mathrm{~m}>\mathrm{h}_{b}>30 \mathrm{~m}$

$G\left(h_{m}\right)=10 \log \left(\mathrm{h}_{m} / 3\right) \mathrm{h}_{m}<=3 \mathrm{~m}$

$G\left(h_{m}\right)=20 \log \left(\mathrm{h}_{m} / 3\right) 10 \mathrm{~m}>\mathrm{h}_{m}>3 \mathrm{~m}$

Figure 3 shows the result of Table 1. The left and middle ones of 3 bars are base parameters, the right one is extended parameter.

\subsubsection{Hata model}

The above model is inconvenient for calculations by a computer. Hata established empirical mathematical relationships to describe the graphical information given by Okumura. Hata's formulation is limited to certain ranges of input parameters and is applicable only over quasi-smooth terrain. To increase the ease of implementation, Hata suggested an empirical model for describing graphical information provided by Okumura. Consequently, the Hata model (a mathematical notation) is also based

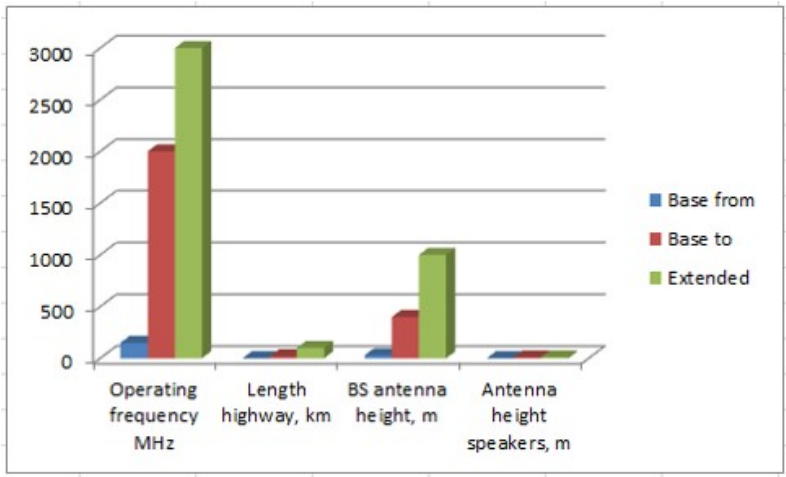

Figure 3. Experimental results of Okumura model.

Table 2. Possible values for the model parameters in the Hata model

\begin{tabular}{|c|c|c|}
\hline \multirow[t]{2}{*}{ Parameters } & \multicolumn{2}{|c|}{$\begin{array}{l}\text { Areas of application of the } \\
\text { Hata model }\end{array}$} \\
\hline & Base & Extended \\
\hline $\begin{array}{l}\text { Operating frequency } \\
(\mathrm{MHz})\end{array}$ & $\begin{array}{l}\text { from } 150 \text { to } \\
1500\end{array}$ & from 150 to 1500 \\
\hline Length highway (km) & $\begin{array}{l}\text { from } 1 \text { to } \\
20\end{array}$ & to 80 \\
\hline BS antenna height (m) & $\begin{array}{l}\text { from } 30 \text { to } \\
200\end{array}$ & from $1 \sim 5$ to 400 \\
\hline $\begin{array}{l}\text { Antenna height } \\
\text { speakers }(\mathrm{m})\end{array}$ & $\begin{array}{l}\text { from } 1 \text { to } \\
10\end{array}$ & from 1 to 10 \\
\hline
\end{tabular}

BS, base station.

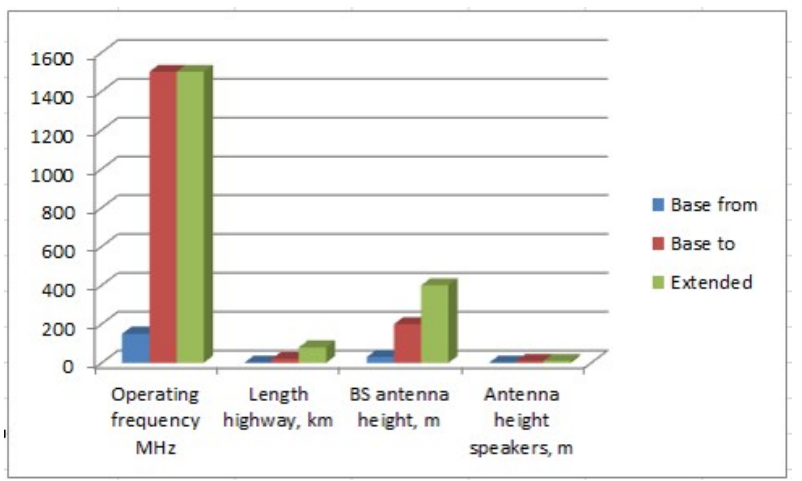

Figure 4. Experimental results of Hata model.

on the experimental data from Okumura. The Hata model is used when changing parameter values within the limits specified in Table 2 and Figure 4.

Figure 4 shows the result of Table 2. The left and middle ones of 3 bars are base parameters, the right one is extended parameter.

Although, the Hata formula does not allow us to take into account all of the specific amendments that are available in the 
method of Okumura, it has significant practical importance.

\subsubsection{Walfisch-Ikegami model}

The Walfisch-Ikegami model is recognized as the best model for predicting signal information in small cells. This model is based on a physical representation of the field at the receiver in the form of two components: a coherent and a scattered component. The coherent component is determined using a wave diffract around the buildings along the road between BSs. The scattered component includes waves that are generated as a result of reconstruction of the incident wave from waves at the BSs. The scattered component includes directions that do not coincide with the direction of the BS and are even opposite to its direction. This model states, that, in a city with relatively low, densely spaced buildings, basic signal propagation (in the absence of direct line of sight [LOS] between nodes) is a path through the roofs of buildings (which can be described as a series of consecutive screens), followed by multiple scattering. The main parameters used in this model: transmission frequency $(f)$, transmitting antenna height $\left(h_{b}\right)$, height of the receiving antenna $\left(h_{m}\right)$, distance between the transmitter and receiver $(d)$, average building height $\left(h_{r}\right)$, average width of the streets $(w)$, and distance between buildings $(b)$. The energy of the received signal depends on where the transmitter and the receiver are located [3].

Calculation formulas for Walfisch-Ikegami model are obtained for the following parameters: distance between the BS and $\mathrm{BS}$ of from $0.02 \mathrm{~km}$ to $5 \mathrm{~km}$ in the frequency range of 800 to $2000 \mathrm{MHz}$, a BS antenna height $h_{t}=4$ to $50 \mathrm{~m}$, height of antennas $h_{r}=1$ to $3 \mathrm{~m}$, and a height of buildings close to BSs of up to $60 \mathrm{~m}$ in Table 3 .

(1) In the LOS. To calculate the propagation loss using a relatively simple formula

(2) Out of sight (none line of sight [NLOS]).

This case is more complicated. The amount by which the received signal strength decreases in passing from the source to the receiver is given by Figure 5 .

The Walfisch-Ikegami model is a pathloss model for the case of small distances between MS and BS, and/or small height of the MS. The total pathloss for the LOS case is given by the equation (8).

$$
P L=42.6+26 \log (d)+20 \log (f c)
$$

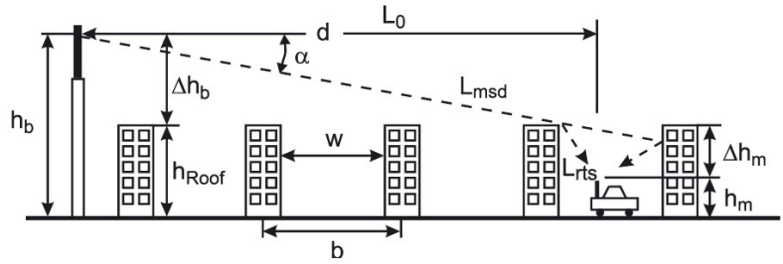

Figure 5. Parameters in the Walfisch-Ikegami model.

For $d>20 \mathrm{~m}$, where again $d$ is in units of kilometers, and $f c$ is in units of MHz. For the NLOS case, the pathloss consists of the free-space pathloss $L 0$, the multiscreen loss $L_{m s d}$ along the propagation path, and the attenuation from the last roofedge to the MS, $L_{r t s}$ (rooptop-to-street diffraction and scatter loss):

PL

$$
=\left\{\left(\begin{array}{cc}
P L 0+L r t s+L m s d & \text { for } L r t s+L m s d>0 \\
P L 0 & \text { for } L r t s+L m s d \leq 0
\end{array}\right)\right\}
$$

The free-space pathloss is the equation (10).

$$
L 0=32.4+20 \log d+20 \log f_{c}
$$

Ikegami derived the diffraction loss $L_{r t s}$ as $L_{r t s}=-16.9-$ $10 \log w+10 \log f_{c}+20 \log h_{m}+$ Lori wherew is the width of the street in meters, and

$$
\Delta h_{m}=h_{\text {Roof }}-h_{m}
$$

is the difference between the building height $h_{\text {Roof }}$ and the height of the MS $h_{m}$.

For the computation of the multiscreen loss $L_{m s d}$, building edges are modeled as screens. The multiscreen loss is then given as

$$
L_{m s d}=L_{b s h}+k_{a}+k_{d} \log d+k_{f} \log f_{c}-9 \log b
$$

$k_{f}$ and $k_{d}$ parameters determine the dependence of the propagation loss of the signal frequency and dispersion when passing through successive screens. Losses increase with decreasing height of the antenna relative to the average height of the surrounding buildings set in parameter $k_{a}$.

When designing small cells, we require information regarding specific areas of city roads. In some cases, one can use the statistics for the city: for a modern urban areas, building density is assumed to be 90 buildings per $\mathrm{km}^{2}$. The buildings' average length is 80 to $105 \mathrm{~m}$; their width is $15 \mathrm{~m}$. Buildings have from 
Table 3. Range of validity for the Walfisch-Ikegami model

\begin{tabular}{lcl}
\hline Carrier frequence & $f_{c}$ & $\begin{array}{l}800 \text { to } 2000 \\
\text { MHZ }\end{array}$ \\
Height of the BS antenna & $h_{b}$ & 4 to $50 \mathrm{~m}$ \\
Height of the MS antenna & $h_{m}$ & 1 to $3 \mathrm{~m}$ \\
Distance & $d$ & 0.02 to $5 \mathrm{~km}$ \\
\hline
\end{tabular}

BS, base station.

9 to 14 floors. The gaps between buildings 15 to $20 \mathrm{~m}$. The average distance of LOS in a layer is $170 \mathrm{~m}$. Garages can be considered homogeneous for areas in which there are no large open parks.

\section{Conclusions}

This study reviewed radio wave propagation models based on BS and antenna characteristics. Each model is described separately, in terms of their characteristics, parameters, and areas of application. The ideal distance at which to use the current model are determined. The model considers the current structure of the BSs. The research shows how these models affect the performance of mobile networks in different scenarios.

\section{Conflict of Interest}

No potential conflict of interest relevant to this article was reported.

\section{Acknowledgements}

This paper was supported by Gachon University in 2015 .

\section{References}

[1] http://www.radioelectronics.com/info/ cellulartelecomms/heterogeneous-networkshetnet/basics-tutorial.php

[2] http://www.ericsson.com/res/thecompany/docs/ publications/ericsson_review/2011/heterogeneous networks.pdf

[3] Varakin L.E. Statistical model of multipath propagation in city, Radiotechnics, no.12, pp.56-61, 1989

[4] Lee W.C.Y. Mobile cellular telecommunications systems. - Howard W. Sam's \&Co., 1989.
[5] Ramona Pinto, LTE: Long Term Evolution. International Journal of Scientific \&Engineering

[6] Yang Yu, Se?n Murphy, Liam Murphy. A Clustering Approach to Planning Base Station and Relay Station Locations in IEEE 802.16j Multi-hop Relay Networks, Department of Computer Science and Informatics University College Dublin, Ireland

[7] Patrice Cal'egari, Fr'ed'eric Guidec, Pierre Kuonen, Frank Nielsen, Combinatorial optimization algorithms for radio network planning, Swiss Federal Institute of Technology, 1015 Lausanne, Switzerland, Ecole Polytechnique, Laboratoire d'informatique LIX, CNRS Unite 143991128 Palaiseau Cedex, France, Accepted April 2000, Theoretical Computer Science, vol. 263, pp. 235-245, 2001.

[8] Altayeva A.B., Omarov B.S., Aitmagambetov A.Z., Kendzhaeva B.B., Burkitbayeva M.A. Modeling and exploring base station characteristics of LTE mobile networks. Life Sci J, vol.11, no.6, pp.227-233, 2014. (ISSN:1097-8135). http://www.lifesciencesite.com.

[9] A.A. Bertossi, M.C. Pinotti, R. Rizzi, \& P. Gupta, "Scalable algorithms for server allocation in infostations", in: Handbook of Research on Scalable Computing Technologies (Co-Editors Kuan-Ching, Ching-Hsien, Laurence, Jack and Hans), IGI Publishing, 2010, ISBN: 978-160566-661-7.

[10] Stojmenovic, Honeycomb networks: topological properties and communication algorithms, 1997.

[11] A.A. Bertossi, M.C. Pinotti, R. Rizzi, \& A.M. Shende "Channel Assighment for Interference Avoidance in Honeycomb Wireless Networks", Journal of Parallel and Distributed Computing. vol.64, no. 12, pp.1329-1344, 2004.

[12] Lei Chen. Coverage Planning and Resource Allocation in Broadband Cellular Access Optimization Models and Algorithms. Linkoping Studies in Science and Technology. Theses no. 1454. Norrkoping University, Sweden, 2010. Lee W.C.Y. Mobile cellular telecommunications systems. - Howard W. Sam's \&Co., 1989. 


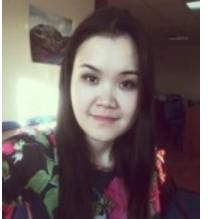

Aigerim Bakatkaliyevna Altayeva received her B.S. and M.Sc., from the Department of Computer Science, MUIT, Kazakhstan, in 2012, and 2014, respectively. Now she is a Ph.D. student at Gachon University. Her areas of interest are AI, Smart Cities, Big Data, and Information Retrieval.

Tel: $+82-31-750-5800$

Fax: +81-32-750-5662

E-mail: aikosha1703@gmail.com

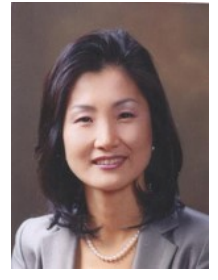

Young Im Cho received her B.S., M.Sc., and Ph.D from the Department of Computer Science, Korea University, Korea, in 1988, 1990, and 1994, respectively. She is a professor at Gachon University. Her areas of interest are AI, Smart Cities, Big Data, and Information

Retrieval.

Tel: +82-31-750-5800

Fax: +81-32-750-5662

E-mail: yicho@gachon.ac.kr 